\title{
Could urinary kidney injury molecule- 1 be a good marker in subclinical acute kidney injury in mild to moderate COVID-19 infection?
}

\author{
Emre Yasar ${ }^{1} \cdot$ Hasan Selcuk Ozger ${ }^{2} \cdot$ Hacı Hasan Yeter ${ }^{1} \cdot$ Cigdem Yildirim $^{2} \cdot$ Zakir Osmanov $^{3} \cdot$ Taha Enes Cetin $^{4} \cdot$ \\ Omer Faruk Akcay ${ }^{1} \cdot$ Neslihan Bukan $^{3}$. Ulver Derici ${ }^{1}$
}

Received: 15 March 2021 / Accepted: 20 June 2021 / Published online: 2 July 2021

(c) The Author(s), under exclusive licence to Springer Nature B.V. 2021

\begin{abstract}
Purpose To evaluate urinary kidney injury molecule-1 (uKIM-1), which is a proximal tubule injury biomarker in subclinical acute kidney injury (AKI) that may occur in COVID-19 infection.

Methods The study included proteinuric $(n=30)$ and non-proteinuric $(n=30)$ patients diagnosed with mild/moderate COVID-19 infection between March and September 2020 and healthy individuals as a control group $(n=20)$. The uKIM-1, serum creatinine, cystatin $\mathrm{C}$, spot urine protein, creatinine, and albumin levels of the patients were evaluated again after an average of 21 days.

Results The median (interquartile range) uKIM-1 level at the time of presentation was 246 (141-347) $\mathrm{pg} / \mathrm{mL}$ in the proteinuric group, 83 (29-217) $\mathrm{pg} / \mathrm{mL}$ in the non-proteinuric group, and $55(21-123) \mathrm{pg} / \mathrm{mL}$ in the control group and significantly high in the proteinuric group than the others $(p<0.001)$. Creatinine and cystatin $\mathrm{C}$ were significantly higher in the proteinuric group than in the group without proteinuria, but none of the patients met the KDIGO-AKI criteria. uKIM-1 had a positive correlation with PCR, non-albumin proteinuria, creatinine, cystatin C, CRP, fibrinogen, LDH, and ferritin, and a negative correlation with eGFR and albumin $(p<0.05)$. In the multivariate regression analysis, non-albumin proteinuria $(p=0.048)$ and BUN ( $p=0.034)$ were identified as independent factors predicting a high uKIM-1 level. After $21 \pm 4$ days, proteinuria regressed to normal levels in $20(67 \%)$ patients in the proteinuric group. In addition, the uKIM-1 level, albuminuria, nonalbumin proteinuria, and CRP significantly decreased.

Conclusions Our findings support that the kidney is one of the target organs of the COVID-19 and it may cause proximal tubule injury even in patients that do not present with AKI or critical/severe COVID-19 infection.
\end{abstract}

Keywords COVID-19 $\cdot$ Urinary kidney injury molecule- $1 \cdot$ Sub-clinical $\cdot$ Acute kidney injury $\cdot$ Non-albuminuric proteinuria

Emre Yasar

rasayerm@hotmail.com

1 Department of Nephrology, Dialysis and Transplantation, Faculty of Medicine, Gazi University, Bahriye Ucok, 06560 Ankara, Turkey

2 Department of Infectious Disease and Clinical Microbiology, Faculty of Medicine, Gazi University, Ankara, Turkey

3 Department of Medical Biochemistry, Faculty of Medicine, Gazi University, Ankara, Turkey

4 Department of Internal Medicine, Faculty of Medicine, Gazi University, Ankara, Turkey

\section{Introduction}

Coronaviruses represent an enveloped RNA virus family, including SARS-CoV settling in epithelial cells, which caused the severe acute respiratory syndrome (SARS) epidemic in 2003, and MERS-CoV, which was responsible for the Middle East Respiratory Syndrome (MERS) epidemic in 2012. As a member of this family, SARS-CoV-2, first identified in December 2019, results in a wide spectrum of disease ranging from asymptomatic to pneumonia manifesting with respiratory distress of varying severity, ultimately leading to multiple organ failure with severe kidney injury [1].

One of the most important organs involved in the disease is the kidney. In cross-sectional studies and meta-analyses 
conducted in China, the United States of America, and Europe, the rate of COVID-19-related acute kidney injury (AKI) varies between 0.5 and $46 \%$ [2-5]. Possible causes of AKI in patients with COVID-19 infection include sepsis and acute tubular necrosis (ATN) caused by renal hypoperfusion, cytokine release syndrome, renal medullary hypoxia secondary to alveolar damage, thrombotic microangiopathy, and rhabdomyolysis [6]. The literature contains data indicating that COVID-19 infection affects the kidney by direct viral invasion, as well as indirect mechanisms [7-9]. Similar to the SARS-CoV infection, in COVID-19, it has been shown that spike protein interacts with angiotensin-converting enzyme 2 (ACE2) as a receptor in the host cell and uses the cellular transmembrane serine proteases family [10]. In the kidneys, it has been reported that ACE2 expressed at a higher rate than in the lungs. ACE2 expression demonstrated in the apical surface of the brush-like edge in all segments of the proximal tubule cells. It is also expressed to a lesser extent in podocytes [11, 12]. Following cell entry, the virus may directly cause kidney cell's injury due to viral cytotoxicity similar to that occurring in lung tissue [13].

Kidney injury molecule-1 (KIM-1) was first defined in 1998 as a type 1 transmembrane protein with an immunoglobulin and mucin domain that is upregulated specifically in the renal proximal tubule [14]. Later, it was shown that in patients with confirmed ATN, intensive KIM-1 expression occurred, especially on the apical surface of proximal tubule cells, which was not detected in glomeruli, and urinary KIM-1 (uKIM-1) was presented as one of the early indicators of tubular damage [15]. It is suggested that there may be a relationship between AKI and KIM-1 in patients with COVID-19 based on the high expression of ACE2 receptors in proximal tubules used by the virus as a cell entry mechanism, demonstration of viral inclusion bodies in the proximal tubule in pathology studies, and the expression of KIM-1 in proximal tubules.

We considered that the uKIM-1 levels of patients with COVID-19 may be affected by the infection causing proximal tubule injury due to a possible direct cytopathic effect and leading to the development of AKI, proteinuria, and/or hematuria. Based on this idea, we conducted this study to contribute to the explanation of the mechanism of renal damage caused by COVID-19 infection, especially in proximal tubules, evaluate the relationship between the uKIM-1 level, kidney injury, and proteinuria, and investigate the course of renal damage and uKIM-1 level in the follow-up of these patients.

\section{Materials and methods}

\section{Patient selection}

The COVID-19 group consisted of 60 patients aged $\geq 18$ years with a definitive diagnosis of
COVID-19 based on the analysis of a nasopharyngeal swab. At the time of presentation, 30 of the patients in this group had $\geq 150 \mathrm{mg} / \mathrm{g}$ proteinuria and the remaining patients had $<150 \mathrm{mg} / \mathrm{g}$ proteinuria. The control group consisted of 18-year-old healthy volunteers without COVID-19 infection according to the polymerase chain reaction (PCR) or serum antibody evaluation by ELISA and no evidence of kidney damage and any known history of kidney disease in previous examinations. As a result, 30 patients with proteinuria, 30 patients without proteinuria, and 20 controls were included in the study. The participants' age, gender, comorbidities, medications, smoking, symptoms, and time elapsed since the onset of symptoms were recorded.

Patients with severe/critical COVID-19 infection, AKI, sepsis/septic shock, rhabdomyolysis, active malignancy, diabetes mellitus, obesity, history of renal transplantation, active use of immunosuppressive drugs, and a history of primary glomerular/tubular diseases at the time of presentation or during the follow-up were excluded from the study.

The severity of COVID-19 disease was determined according to the WHO criteria [16]. Procalcitonin $>0.5 \mathrm{mg} /$ $\mathrm{dl}$ was considered significant in addition to the parameters specified in the WHO COVID-19 severity criteria in terms of sepsis/septic shock. In the exclusion of patients, AKI and chronic kidney disease (CKD) were defined according to the KDIGO criteria $[17,18]$. An albumin/creatinine ratio (ACR) of $>30 \mathrm{mg} / \mathrm{g}$ was accepted to indicate the presence of albuminuria $\left(\mathrm{A}_{2}\right)$, and a protein/creatinine ratio (PCR) of $>150 \mathrm{mg} / \mathrm{g}$ was defined as proteinuria. The estimated glomerular filtration rate (eGFR) was obtained using the CKD-EPI creatinine-cystatin $\mathrm{C}$ formula.

\section{Laboratory parameters}

At presentation, complete blood count (CBC), C-reactive protein (CRP), blood urea nitrogen (BUN), creatinine, cystatin $\mathrm{C}$, uric acid, albumin, alanine aminotransferase (ALT), aspartate aminotransferase, (AST), lactate dehydrogenase $(\mathrm{LDH})$, creatine kinase $(\mathrm{CK})$, D-dimer, ferritin, fibrinogen, complete urinalysis, spot urine protein, creatinine, and albumin were studied. CBC, CRP, BUN, creatinine, cystatin $\mathrm{C}$, uric acid, albumin, complete urinalysis, spot urine protein, creatinine, and albumin were examined again within an average of 21 days. Urine samples were taken from the first urine in the morning. Non-albumin proteinuria was calculated by subtracting urinary ACR (uACR) from urinary PCR (UPCR). Serum cystatin C, urinary albumin, protein and creatinine, albümin, uric acid, CRP, CK, LDH, ALT, and AST levels were measured by Beckman-Coulter (US) AU5800 Clinical Chemistry autoanalyzer using original kits. Ferritin levels were determined with a Beckman Coulter (US) DxI800 Access Immunoassay System. Urine erythrocyte analysis was perforrmed using the Sysmex (Jp) UF 
5000 analyzer. D-dimer and fibrinogen levels were measured using the Stago (Fr) STA Compact Max3 analyzer and Hemoglobin, White blood cell, lymphocyte, neutrophile and platelet measurement were perfomed using the Sysmex (Jp) XN 5000 analyzer.

Urine samples were taken into sterile containers for the measurement of the uKIM-1 level. The samples of the patients were centrifuged at $3500 \mathrm{rpm}$ for $10 \mathrm{~min}$ before storage and separated from the sediment. After centrifugation, the samples were transferred to Eppendorf tubes and stored at $-80{ }^{\circ} \mathrm{C}$ until analysis. The uKIM- 1 level was measured with the ELISA method. During this analysis, washing procedures were performed with a Biotek device (ELx50 Bioelisa Washer, Bio-Tec.), and absorbance reading using a Biotek reader (ELx 800 UV Universal Microplate Reader, Bio-Tec. Instruments, Inc). The measurement of the uKIM-1 levels was undertaken with the sandwich ELISA method using a USCN-Cloud human KIM-1 ELISA kit (Product no: SEA785Hu Lot no: L200831605). The lower measurement limit of the kit was less than $32 \mathrm{pg} / \mathrm{mL}$, the measurement range was $78-5,000 \mathrm{pg} / \mathrm{mL}$, and the intra-run and interrun coefficient of variation values were $<10 \%$ and $<12 \%$, respectively. The samples belonging to the patients in the study were carried out by considering the instructions in the kit prospectus. Absorbances of the standard and samples were measured at 450-nm wavelength. Concentrations in the samples were calculated using the formula obtained from the standard curve drawn with the concentrations and absorbance values of the standards (GraphPad software). The results obtained were expressed as $\mathrm{pg} / \mathrm{mL}$.

\section{Statistical analysis}

Statistical analyses were performed using SPSS v. 20. The normality of numerical data was evaluated with the Shapiro-Wilk test. Parametric data were given as mean \pm standard deviation, non-parametric data as median (interquartile range), and categorical data as frequency (percentage). Nonparametric data of more than two groups were compared with the Kruskal-Wallis test, and paired comparision of the groups were also undertaken with the Mann-Whitney $U$ test. Analysis of variance was used to compare the parametric data of more than two groups. Groups that resulted in statistical differences were determined using the post hoc Tukey analysis. Student's $t$ test was conducted for the comparison of parametric data of two independent groups, and Pearson's chi-squared or Fisher's exact test was used for the comparison of categorical data. For numerical data, a correlation analysis was performed. The receiver operating characteristic (ROC) analysis was carried out to determine the optimum cut-off values at which numerical data could predict a categorical variable. In addition, the multivariate logistic regression analysis was employed to determine independent predictive factors predicting a high uKIM-1 level. A $p$ value of $<0.05$ was considered statistically significant.

\section{Results}

Twenty (67\%) of the patients in the proteinuric group, 18 $(60 \%)$ of those in the non-proteinuric group, and $12(60 \%)$ controls were male. There was no difference in age, gender, comorbidities, and smoking status between the three groups. Pneumonia was present in $20(67 \%)$ patients in the proteinuric and $14(47 \%)$ patients in the non-proteinuric group at admission $(p=0.091)$. The median time from the onset of symptoms to presentation was $3(1-5)$ days in the proteinuric group and $0(0-3)$ days in the non-proteinuric group $(p=0.010)$. When the patients with and without proteinuria were compared, CRP, ferritin, D-dimer, fibrinogen, and LDH were higher in the proteinuric group $(p<0.001$ for all). In addition, lymphocyte and platelet counts were significantly lower in the proteinuric group $(p<0.001$ and $p=0.034$, respectively). The general characteristics of the patients, their clinical and laboratory findings at presentation, and treatments they received for COVID-19 are shown in Table 1.

The median (IQR) uKIM-1 level at presentation was 246 (141-347) $\mathrm{pg} / \mathrm{mL}$ in the proteinuric group, 83 (29-217) pg/ $\mathrm{mL}$ in the non-proteinuric group, and $55(21-123) \mathrm{pg} / \mathrm{mL}$ in the control group. uKIM-1 was significantly higher in the proteinuric patients compared to both the patients without proteinuria and controls $(p<0.001)$. The median uPCR of the proteinuric group was measured as 309 (IQR: 250-558) $\mathrm{mg} / \mathrm{g}$. When proteinuria was further analyzed, it was determined that most cases presented with non-albumin protein [median 275 (IQR: 215-445) mg/g]. Albuminuria $\left(\mathrm{A}_{2}\right)$, was present in $19(63 \%)$ of the patients in the proteinuric group and four (13\%) of those in the non-proteinuric group. Creatinine and cystatin $\mathrm{C}$ were significantly higher in the proteinuric group than in the group without proteinuria, but none of the patients met the KDIGO-AKI criteria. The results of the renal function tests and comparison between the groups are shown in Table 2 and Fig. 1A.

The median (IQR) uKIM-1 level at presentation was $224(120-273) \mathrm{pg} / \mathrm{mL}$ in patients with pneumonia and 100 (37-287) $\mathrm{pg} / \mathrm{mL}$ in patients without pneumonia. However, this difference was not statistically significant $(p=0.112)$. Proteinuria was found to be $259(105-465) \mathrm{mg} / \mathrm{g}$ in patients with pneumonia and $94(65-240) \mathrm{mg} / \mathrm{g}$ in patients without pneumonia $(p=0.019)$ (data not shown).

In the correlation analysis of the laboratory parameters of all participants at admission, uKIM-1 had a positive correlation with PCR $(r=0.355, p=0.002)$, non-albumin proteinuria $(r=0.398, p<0.001)$, creatinine $(r=0.285$, $p=0.013)$, cystatin C $(r=0.411, p<0.001), \mathrm{CRP}(r=0.376$, 
Table 1 Characteristics, clinical findings and laboratory parameters of patients at the time of presentation

\begin{tabular}{|c|c|c|c|c|}
\hline Variables & $\begin{array}{l}\text { Proteinuric } \\
(n=30)\end{array}$ & $\begin{array}{l}\text { Nonproteinuric } \\
(n=30)\end{array}$ & $\begin{array}{l}\text { Control } \\
(n=20)\end{array}$ & $p$ value \\
\hline Age, mean, SD & $45.0 \pm 10.5$ & $43.1 \pm 11.4$ & $44.1 \pm 10.1$ & 0.769 \\
\hline \multicolumn{5}{|l|}{ Gender, $n(\%)$} \\
\hline Male & $20(67)$ & $18(60)$ & $12(60)$ & 0.879 \\
\hline \multicolumn{5}{|l|}{ Comorbidities, $n(\%)$} \\
\hline Chronic hypertension & $4(13)$ & $3(10)$ & $3(15)$ & 0.882 \\
\hline Chronic heart disease & $1(3)$ & 0 & $1(5)$ & 0.516 \\
\hline Asthma & $1(3)$ & $3(10)$ & $2(10)$ & 0.534 \\
\hline \multicolumn{5}{|l|}{ Medicines used at home, $n(\%)$} \\
\hline ACE inhibitors & $1(3)$ & $2(7)$ & $1(5)$ & 0.823 \\
\hline $\mathrm{ARB}$ & $1(3)$ & $1(3)$ & $1(5)$ & 0.952 \\
\hline Other anti-hypertensives & $2(7)$ & 0 & $1(5)$ & 0.387 \\
\hline Current smoker, $n(\%)$ & $3(10)$ & $3(10)$ & $3(15)$ & 0.965 \\
\hline \multicolumn{5}{|l|}{ COVID-19 severity, $n(\%)$} \\
\hline Mild & $10(33)$ & $16(53)$ & & 0.091 \\
\hline Moderate & $20(67)$ & $14(47)$ & & \\
\hline Symptom duration (days), median IQR (25-75) & $3(1-5)$ & $0(0-3)$ & & 0.010 \\
\hline \multicolumn{5}{|l|}{ Symptoms $n(\%)$} \\
\hline Fever $>38^{\circ} \mathrm{C}$ & $8(27)$ & $4(13)$ & & 0.219 \\
\hline Cough & $17(57)$ & $10(33)$ & & 0.047 \\
\hline Dyspnea & $12(40)$ & $6(20)$ & & 0.103 \\
\hline Headache & $5(17)$ & 0 & & 0.022 \\
\hline Sore throat & $4(13)$ & $3(10)$ & & 0.723 \\
\hline Myalgia and arthralgia & $6(20)$ & $3(10)$ & & 0.302 \\
\hline Diarrhea & $2(7)$ & $2(7)$ & & 0.972 \\
\hline \multicolumn{5}{|l|}{ Treatment, $n(\%)$} \\
\hline Favipiravir & $15(50)$ & $5(17)$ & & 0.008 \\
\hline HQ & $13(43)$ & $24(80)$ & & 0.002 \\
\hline HQ + favipiravir & $2(7)$ & $1(3)$ & & 0.157 \\
\hline \multicolumn{5}{|l|}{ Laboratory parameters, median IQR (2575) } \\
\hline $\mathrm{CRP}(\mathrm{mg} / \mathrm{dL})^{\mathrm{a}}$ & $50(15.0-126.2)$ & $4,7(1.9-11.1)$ & $1.6(1.4-2.4)$ & $<0.001$ \\
\hline Ferritin $(\mathrm{ng} / \mathrm{mL})^{\mathrm{a}}$ & $288(128-755)$ & $66(29-108)$ & $56(27-80)$ & $<0.001$ \\
\hline $\mathrm{D}$-dimer $(\mu \mathrm{g} / \mathrm{mL})^{\mathrm{a}}$ & $0.40(0.27-0.69)$ & $0.21(0.16-0.32)$ & $0,23(0.16-0.29)$ & $<0.001$ \\
\hline Fibrinogen $(\mathrm{mg} / \mathrm{dl})^{\mathrm{a}}$ & $461(342-564)$ & $297(237-330)$ & $282(235-315)$ & $<0.001$ \\
\hline CK (U/L) & $103(79-180)$ & $98(74-125)$ & $56(46-69)$ & $<0.001$ \\
\hline $\mathrm{LDH}(\mathrm{U} / \mathrm{L})^{\mathrm{a}}$ & $284(236-367)$ & $203(177-244)$ & $210(184-223)$ & $<0.001$ \\
\hline $\operatorname{ALT}(\mathrm{U} / \mathrm{L})$ & $31(23-43)$ & $26(15-31)$ & $19(15-33)$ & 0.051 \\
\hline $\operatorname{AST}(\mathrm{U} / \mathrm{L})^{\mathrm{a}}$ & $36(27-46)$ & $22(19-28)$ & $23(16-29)$ & $<0.001$ \\
\hline HGB (g/dL) & $14.1(12.8-14.6)$ & $14.4(13.0-15.4)$ & $15.2(13.8-16.1)$ & 0.215 \\
\hline $\mathrm{WBC}\left(\mathrm{mm}^{3}\right)$ & $5905(4712-7680)$ & $6750(5180-7685)$ & 6895 (5685-8777) & 0.271 \\
\hline Neutrophil count $\left(\mathrm{mm}^{3}\right)$ & $4050(2705-5657)$ & $3560(3010-5255)$ & $3655(3135-5010)$ & 0211 \\
\hline Lymphocyte count $\left(\mathrm{mm}^{3}\right)^{\mathrm{a}}$ & $1170(935-1582)$ & $1870(1515-2180)$ & $2230(1842-2840)$ & $<0.001$ \\
\hline Platelet count $\left(\times 10^{3} \mathrm{~mm}^{3}\right)^{\mathrm{a}}$ & $221(157-252)$ & $242(217-299)$ & $250(219-283)$ & 0.003 \\
\hline
\end{tabular}

Bold values are statistically significiant

$A C E$ angiotensin-converting enzyme; $A R B$ angiotensin receptor blocker; $H Q$ hydroxychloroquine, $C R P$ C-reactive protein; $A L T$ alanine aminotransferase; $A S T$ aspartate aminotransferase, $L D H$ lactate dehydrogenase; $C K$ creatine kinase, $H G B$ hemoglobin, $W B C$ white blood cell, $I Q R$ inter-quartile range; $S D$ Standard deviation

${ }^{\mathrm{a}}$ When the patients with and without proteinuria were compared, the $p$ value was $<0.05$ 
Table 2 Renal functions, CRP levels and lymphocyte counts of the patients at presentation and during follow-up (mean 21 days after presentation)

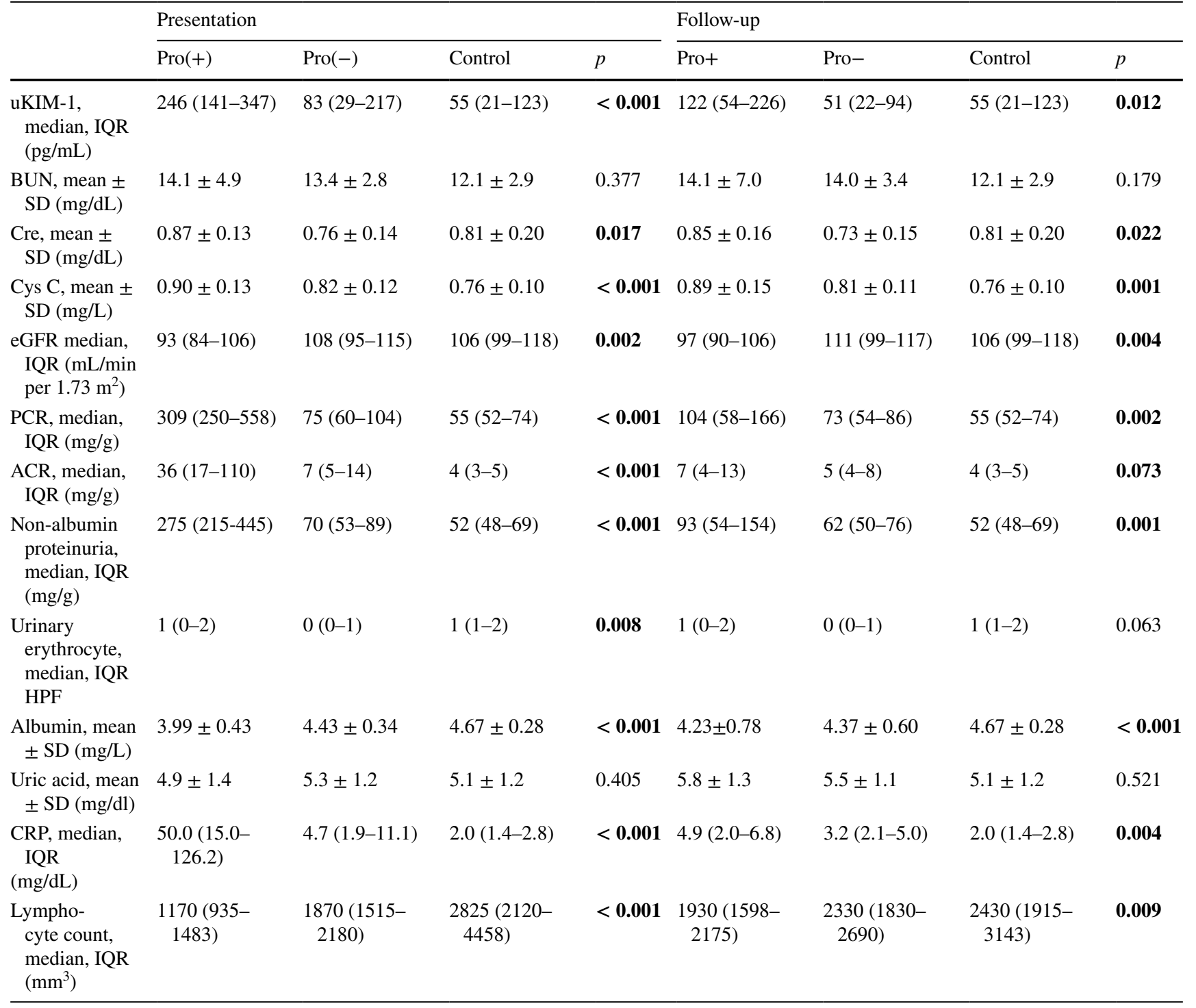

Bold values are statistically significiant

uKIM-1 urinary kidney injury molecule-1; BUN blood urea nitrogen; Cre creatinine; $C y s$ C Cystatin C; $e G F R$ estimated glomerular filtration rate; $P C R$ protein/creatinine ratio; $A C R$ albumin/creatinine ratio; $H P F$ high power field; $C R P$ C-reactive protein; Pro(+)proteinuric group; $P r o(-)$ non-proteinuric group, IQR inter-quartile range; $S D$ standard deviation

$p<0.001)$, fibrinogen $(r=0.443, p<0.001)$, LDH $(r=0.281$, $p=0.014)$ and ferritin $(r=0.336, p=0.003)$ and a negative correlation with eGFR $(r=-0.334, p=0.003)$ and albu$\min (r=-0.346, p=0.002)$. PCR was found to be positively correlated with non-albumin proteinuria $(r=0.980$, $p<0.001)$, cystatin C $(r=0.318, p=0.014), \mathrm{CRP}(r=0.457$, $p<0.001)$, fibrinogen $(r=0.513, p<0.001), \mathrm{LDH}(r=0.468$, $p<0.001)$ and ferritin $(r=0.415, p<0.001)$ and negatively correlated with eGFR $(r=-0.297, p=0.008)$ and albumin $(r=-0.507, p<0.001)$. The results of the correlation analysis are shown in Supplemental Table 1.
The ROC analysis on the data of all participants revealed a cut-off value of $145 \mathrm{pg} / \mathrm{mL}$ for uKIM-1 in predicting proteinuria (AUC: 0.830 , sensitivity $77 \%$, and specificity $76 \%$; $p<0.001)$. Using this cut-off value, the participants were divided into two groups as those with low and high uKIM-1 levels. When the laboratory results of the participants with high and low uKIM-1 levels were compared, those with high uKIM-1 levels were determined to have higher PCR, ACR, non-albumin proteinuria, BUN, creatinine, cystatin $\mathrm{C}$, uric acid, CRP, ferritin, fibrinogen, D-dimer and LDH values and lower eGFR, albumin, lymphocyte and platelet values. 

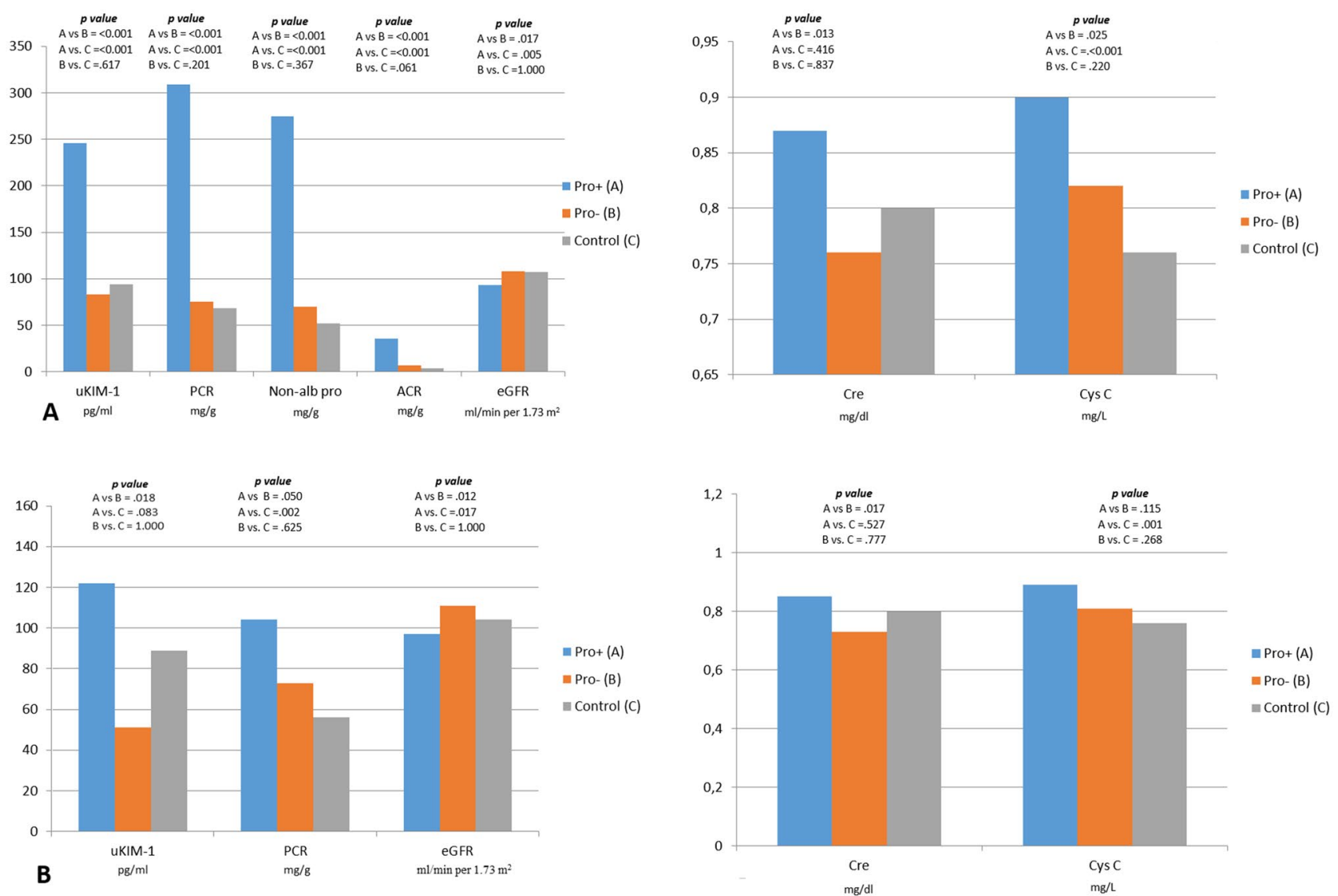

Fig. 1 Comparison results of renal function indicators between the groups at the time of presentation and during the follow-up. A Comparison results of renal function indicators between the groups at the time of presentation. $u K I M-1$ urinary kidney injury molecule-1; $P C R$ protein/creatinine ratio; Non-alb pro non-albumin proteinuria; $A C R$ albumin/creatinine ratio; $e G F R$ estimated glomerular filtration

rate; Pro (+) proteinuric group; Pro (-) non-proteinuric group; $\mathrm{Cre}$ creatinine; Cys $C$ Cystatin C. B Comparison results of renal function indicators between the groups during the follow-up. uKIM-1 urinary kidney injury molecule-1; $P C R$ protein creatinine ratio; $e G F R$ estimated glomerular filtration rate; Pro (+) proteinuric group; Pro (-) non-proteinuric group; $C r e$ creatinine; $C y s$ Cystatin C

Table 3 presents the results of the comparison between the high and low uKIM-1 groups.

To identify factors that independently predict high uKIM-1 level, the multivariate regression analysis was conducted with the PCR, ACR, non-albumin proteinuria, BUN, creatinine, cystatin $\mathrm{C}$, uric acid, CRP, ferritin, fibrinogen, D-dimer, LDH, eGFR, albumin, lymphocyte, and platelet values. According to the results, non-albumin proteinuria $[\mathrm{OR}(95 \% \mathrm{CI})=1.141(1.001-1.301)$, $p=0.048]$ and BUN [OR $(95 \% \mathrm{CI})=1.219(1.016-1.464)$, $p=0.034]$ were found to be independent factors predicting a high uKIM-1 level (Shown in Supp. Table 2).

The patients were re-evaluated after an average of 21 $( \pm 4)$ days. Proteinuria improved in $20(67 \%)$ patients in the proteinuric group. In addition, the uKIM-1 level, albuminuria, non-albumin proteinuria, and CRP significantly decreased. In the non-proteinuric group, the uKIM-1 level

significantly decreased and the values of albuminuria and non-albumin proteinuria also decreased within normal limits. The follow-up results of the patients are shown in detail in Table 2, Supplemental Table 3, and Fig. 1B.

\section{Discussion}

In this study, we found that although the patients with mild and moderate COVID-19 did not meet the AKI criteria, they presented with findings suggesting proximal tubule damage, especially non-albumin proteinuria, and their uKIM-1 levels were increased. In addition, we determined that non-albumin proteinuria and high BUN level were independent factors predicting high uKIM-1 level.

Acute tubular injury has been reported to be the most common pathological finding in biopsy and autopsy series 
Table 3 Results of the laboratory parameters of the participants with high and low uKIM-1 levels at the time of presentation

\begin{tabular}{lllr}
\hline & uKIM-1 & & \\
\cline { 2 - 4 } & Low $(<145)$ & High $(\geq 145)$ & $p$ value \\
\hline PCR, median, IQR $(\mathrm{mg} / \mathrm{g})$ & $75(55-122)$ & $267(99-392)$ & $<\mathbf{0 . 0 0 1}$ \\
ACR, median, IQR $(\mathrm{mg} / \mathrm{g})$ & $5(4-21)$ & $18(7-63)$ & $<\mathbf{0 . 0 0 1}$ \\
Non-albumin proteinuria, median, IQR & $72(52-101)$ & $213(87-363)$ & $<\mathbf{0 . 0 0 1}$ \\
$\quad(\mathrm{mg} / \mathrm{g})$ & & & \\
BUN, mean $\pm \mathrm{SD},(\mathrm{mg} / \mathrm{dl})$ & $12.3 \pm 2.8$ & $14.6 \pm 4.5$ & $\mathbf{0 . 0 2 4}$ \\
Creatinine, mean $\pm \mathrm{SD},(\mathrm{mg} / \mathrm{dL})$ & $0.77 \pm 0.16$ & $0.84 \pm 0.12$ & 0.068 \\
Cystatin C, mean $\pm \mathrm{SD},(\mathrm{mg} / \mathrm{L})$ & $0.79 \pm 0.13$ & $0.89 \pm 0.12$ & $\mathbf{0 . 0 0 1}$ \\
eGFR median, IQR & $104(96-118)$ & $99(85-114)$ & $\mathbf{0 . 0 4 1}$ \\
$\left(\mathrm{ml} / \mathrm{min}\right.$ per $\left.1.73 \mathrm{~m}{ }^{2}\right)$ & & & 0.096 \\
Uric acid, mean $\pm \mathrm{SD}(\mathrm{mg} / \mathrm{dL})$ & $4.9 \pm 1.1$ & $5.3 \pm 1.4$ & $\mathbf{0 . 0 0 6}$ \\
Albumin, mean $\pm \mathrm{SD}(\mathrm{mg} / \mathrm{L})$ & $4.4 \pm 0.4$ & $17.5(4.8-71.0)$ & $\mathbf{0 . 0 0 1}$ \\
CRP, median, IQR $(\mathrm{mg} / \mathrm{dL})$ & $2.5(1.5-9.7)$ & $152(60-429)$ & $\mathbf{0 . 0 1 6}$ \\
Ferritin, median, IQR $(\mathrm{ng} / \mathrm{mL})$ & $69(29-141)$ & $344(316-517)$ & $\mathbf{0 . 0 0 5}$ \\
Fibrinogen, median, IQR $(\mathrm{mg} / \mathrm{dL})$ & $301(245-347)$ & $0.33(0.24-0.65)$ & $\mathbf{0 . 0 0 5}$ \\
D-dimer, median, IQR $(\mu \mathrm{g} / \mathrm{mL})$ & $0.24(0.18-0.32)$ & $260(203-340)$ & $\mathbf{0 . 0 2 2}$ \\
LDH, median, IQR $(\mathrm{U} / \mathrm{L})$ & $213(183-254)$ & $1743 \pm 1390$ & $\mathbf{0 . 0 0 5}$ \\
Lymphocyte, median, IQR $\left(\mathrm{mm}{ }^{3}\right)$ & $2237 \pm 1226$ & $242 \pm 115$ & $\mathbf{0 . 0 3 7}$ \\
Platelet median, IQR $\left(\times 10^{3} \mathrm{~mm}^{3}\right)$ & $261 \pm 70$ & & \\
\hline Bold & & &
\end{tabular}

Bold values are statistically significiant

uKIM-1 urinary kidney injury molecule-1; BUN blood urea nitrogen; eGFR estimated glomerular filtration rate; $P C R$ protein/creatinine ratio; $A C R$ albumin/creatinine ratio; $C R P$ C-reactive protein; $L D H$, lactate dehydrogenase; $I Q R$ inter-quartile range; $S D$ standard deviation performed in patients with COVID-19 infection [7-9, 19, 20]. While two studies did not show exact viral particles matching the definition of coronavirus morphology, in a study including the autopsy evaluation of 26 patients, viral inclusions were observed in the proximal tubules of seven patients $[7,19,20]$. In two other studies, SARS-CoV RNA was isolated from kidney tissue [8,9]. In another study, the urine sample of one patient infected with SARS-CoV-2 was positive for SARS-CoV-2 RNA [21]. In light of these findings, it is considered that COVID-19 infection may cause AKI through indirect mechanisms such as sepsis and ischemia, as well as through a direct cytopathic effect.

During the pandemic period, it has been shown that 56.9\% of hospitalized COVID-19 positive patients and $37.2 \%$ of COVID-19 negative patients developed AKI [22]. There are also studies reporting that proteinuria $(42.1-43.9 \%)$ and hematuria (11.3-40.9\%), which are other indicators of kidney injury, are observed in patients with COVID-19 [23, 24]. In addition, a meta-analysis showed that proteinuria and hematuria could occur without AKI in patients with COVID19. In this meta-analysis, although the rate of patients with increased serum creatinine levels was $9.6 \%$, proteinuria was found in $57.2 \%$ of all patients [25]. However, COVID19 RNA was also detected in the kidney tissue of patients without AKI [9]. In the current study, none of the patients had AKI according to the KDIGO-AKI criteria. However, when the patients were evaluated in terms of the presence of proteinuria, which is an indicator of kidney injury, those with proteinuria had higher UKIM-1, creatinine, and cystatin $\mathrm{C}$ levels than those without proteinuria. In the literature, an AKI subgroup defined as subclinical AKI, which does not meet the conventionally used criteria for the diagnosis of AKI but presents high levels of tubular injury markers, has been reported and suggested to indicate possible kidney injury [26]. One of these markers is uKIM-1. Various studies provide data showing that the uKIM-1 level measured in patients that have not developed AKI could be a marker indicating early kidney injury, as well as a parameter that predicts both the need for renal replacement therapy and mortality in the long term [27-29]. In the current study, the patients with mild/moderate COVID-19 were evaluated, and it was determined that there was a group that could be interpreted as subclinical AKI despite not meeting the KDIGOAKI criteria, which presented with significantly increased proteinuria accompanying high uKIM-1 levels.

It was previously shown that uKIM-1, a tubular biomarker specific to proximal tubule injury, correlates with proteinuria even in trace amounts. In addition, no relationship was found between uKIM-1 and hematuria and pyuria [30]. In another study, it was reported that the level of uKIM-1 increased in proportion to the severity of histological damage in patients with acute tubular injury, and this was positively associated with proteinuria and negatively associated with eGFR [31]. In our study, consistent with the literature, uKIM-1 was 
found to be positively associated with proteinuria and nonalbumin proteinuria, and negatively associated with eGFR.

Acute tubular necrosis being the most common pathological finding in biopsy-autopsy series in COVID-19 patients and intense ACE2 expression in the kidney, especially in proximal tubules suggest that patients with COVID-19 will especially present with proximal tubular injury. A study conducted in a subgroup of patients with severe COVID-19 infection, low molecular weight proteinuria, aminoaciduria, hypouricemia, and hypophosphatemia were found, and proximal tubular dysfunction was reported in the early course of the disease. It was also determined that proximal tubule dysfunction was independent of pre-existing comorbidities, glomerular proteinuria, use of nephrotoxic drugs, and viral load [32]. In the postmortem series of cases who died due to COVID-19, glucosuria, hypokalemia, and hypophosphatemia, which are indicators of possible proximal tubular injury, were observed in a minority of patients, and glomeruli were well preserved in most of the kidneys [19]. In another series of mild/moderate COVID-19 patients, it was shown that alpha-1 macroglobulin, sodium, and potassium excretion increased, suggesting possible proximal tubule injury [33]. In our study, uKIM-1, a specific marker of proximal tubule injury, increased in patients with proteinuria. In addition, most of the protein excreted was found to be nonalbumin. Thus, our study shows that even mild and moderate COVID-19 cases may have proximal tubular injury. To the best of our knowledge, our study is the first in the literature to show that proximal tubule injury can occur even in mildmoderate COVID-19 cases by evaluating the UKIM-1 level.

In our study, we found higher ferritin, CRP, D-dimer, $\mathrm{LDH}$, and fibrinogen and lower lymphocytes and platelet values in the proteinuric group compared to the remaining two groups. In addition, ferritin, CRP, LDH, and fibrinogen were positively correlated with uKIM-1, proteinuria, nonalbumin proteinuria, creatinine, and cystatin $\mathrm{C}$, and negatively correlated with eGFR. Systemic inflammation markers such as ferritin, CRP, and LDH have been reported to be higher in COVID-19 cases presenting with AKI compared to those with normal renal functions [34]. It has also been shown that higher levels of ferritin, D-dimer, fibrinogen, CRP, and LDH are present in patients with AKI [22, 24]. Our findings suggest that systemic inflammation may also contribute to subclinical kidney injury in mild/moderate COVID-19 cases that do not meet the AKI criteria.

In our study, we found that $67 \%$ of the patients in the proteinuric group regressed to the normal ranges within an average of 21 days. We also showed that UKIM-1 and proteinuria regressed in both proteinuric and non-proteinuric groups. In one of the most comprehensive studies conducted on this subject, it was reported that $68.5 \%$ of patients experienced proteinuria remission within 3 weeks after the onset of symptoms. In the same study, patients with moderate
COVID-19 disease had 4.08 times higher remission rate in proteinuria compared to critical disease [35]. In another study including the follow-up results, $65 \%$ of survivors with AKI were observed to have returned to their baseline renal function at the time of discharge. It was shown that of the patient that did not have improved renal functions at discharge, $36 \%$ recovered these functions within a median duration of 21 days [3]. In a smaller study, it was reported that of patients who survived following AKI development, 64\% had improved renal functions evaluated on the 21 st day of follow-up [36]. There is a need for comprehensive studies to further investigate the effect of COVID-19 infection and the resulting proteinuria on long-term renal functions.

The limitations of our study include the small sample size due to the naive patient group selection (excluded common diseases such as DM and obesity), patients experiencing difficulties in attending follow-up due to the quarantine rules and ongoing pandemia. In addition, the number of COVID19 patients decreased in a part of the period we conducted the study. Another limitation may be that the control group consisted of healthy volunteers only. It would be beneficial to establish a second group of patients with non-COVID viral diseases. But influenza and other common viral pathogens that can cause pneumonia were less common due to social distancing, hand disinfection, and the use of masks. That is why creating a such patient cohort was not possible. In addition, the inability to confirm the present findings histopathologically is another important study limitation. Therefore it is difficult mention about the direct viral cythopathic effect on the kidney. However, our study also has certain strengths, such as the exclusion of most possible pathologies that may affect kidney injury, inclusion of only mild/moderate COVID-19 cases, and the uKIM-1 level being evaluated both at presentation and during follow-up, not based on a single measurement.

Our findings show that the kidney is one of the target organs of the SARS-CoV-2 virus and may cause proximal tubule injury even in patients without AKI and critical/ severe COVID-19 infection. We also found that a specific proximal tubule injury biomarker, uKIM-1, was elevated in mild-moderate COVID-19 patients, along with the nonspecific kidney injury markers ACR and PCR. In the course of the disease, increased UKIM 1 and BUN levels and the presence of non-albumin proteinuria may be an indicator of renal tubular injury. Therefore, we recommend that patients with non-albumin proteinuria and high BUN levels should be followed up closely for kidney injury. On the other hand, although the majority of our patients' proteinuria regressed within 3 weeks, further studies with a longer follow-up are needed to determine the possible long-term effects of kidney injury in patients with COVID-19 infection. 
Supplementary Information The online version contains supplementary material available at https://doi.org/10.1007/s11255-021-02937-0.

Author contributions E.Y.: writing, study design and statistical analysis H.S.O: critical review, H.H.Y.: data analysis and design C.Y.: Data collection, Z.O.: laboratory parameters analysis and interpretation T.E.C: data collection, O.F.A: data collection and design N.B.: laboratory parameters analysis and interpretation, U.D.: critical review and drafting article.

Funding Turkish Society of Hypertension and Renal Diseases.

\section{Declarations}

Conflict of interest All the authors have declared no competing interest.

Ethical approval This prospective case-control study was conducted in a single center with patients diagnosed with COVID-19 between March 31, 2020 and September 30, 2020. Informed consent was obtained from all the patients with COVID-19 and controls. The study protocol was approved by the Clinical Research Ethics Committee of our university (approval number: 601 date: 05.10.2020).

\section{References}

1. Epidemiology Working Group for NCIP Epidemic Response, Chinese Center for Disease Control and Prevention (2020) The epidemiological characteristics of an outbreak of 2019 novel coronavirus diseases (COVID-19) in China. Zhonghua Liu Xing Bing Xue Za Zhi 41(2):145-151

2. Guan WJ, Ni ZY, Hu Y, Liang WH, Ou CQ, He JX et al (2020) Clinical characteristics of Coronavirus disease 2019 in China. N Engl J Med 382(18):1708-1720

3. Chan L, Chaudhary K, Saha A, Chauhan K, Vaid A, Zhao S et al (2021) AKI in hospitalized patients with COVID-19. J Am Soc Nephrol 32(1):151-160

4. Regina J, Papadimitriou-Olivgeris M, Burger R, Le Pogam MA, Niemi T, Filippidis P et al (2020) Epidemiology, risk factors and clinical course of SARS-CoV-2 infected patients in a Swiss university hospital: an observational retrospective study. PLOS ONE 15(11):e0240781

5. Robbins-Juarez SY, Qian L, King KL, Stevens JS, Husain SA, Radhakrishnan J et al (2020) Outcomes for patients with COVID19 and acute kidney injury: a systematic review and meta-analysis. Kidney Int Rep 5(8):1149-1160

6. Hassanein M, Radhakrishnan Y, Sedor J, Vachharajani T, Vachharajani VT, Augustine J et al (2020) COVID-19 and the kidney. Cleve Clin J Med 87(10):619-631

7. Su H, Yang M, Wan C, Yi LX, Tang F, Zhu HY et al (2020) Renal histopathological analysis of 26 postmortem findings of patients with COVID-19 in China. Kidney Int 98(1):219-227

8. Puelles VG, Lütgehetmann M, Lindenmeyer MT, Sperhake JP, Wong MN, Allweiss L et al (2020) Multiorgan and renal tropism of SARS-CoV-2. N Engl J Med 383(6):590-592

9. Braun F, Lütgehetmann M, Pfefferle S, Wong MN, Carsten A, Lindenmeyer MT et al (2020) SARS-CoV-2 renal tropism associates with acute kidney injury. Lancet 396(10251):597-598

10. Wu A, Peng Y, Huang B, Ding X, Wang X, Niu P et al (2020) Genome composition and divergence of the novel
Coronavirus (2019-nCoV) originating in China. Cell Host Microbe 27(3):325-328

11. Ye M, Wysocki J, William J, Soler MJ, Cokic I, Batlle D (2006) Glomerular localization and expression of Angiotensin-converting enzyme 2 and Angiotensin-converting enzyme: implications for albuminuria in diabetes. J Am Soc Nephrol 17(11):3067-3075

12. Chen QL, Li JQ, Xiang ZD, Lang Y, Guo GJ, Liu ZH (2020) Localization of cell receptor-related genes of SARS-CoV-2 in the kidney through single-cell transcriptome analysis. Kidney Dis (Basel) 6(4):258-270

13. Xu Z, Shi L, Wang Y, Zhang J, Huang L, Zhang C et al (2020) Pathological findings of COVID-19 associated with acute respiratory distress syndrome. Lancet Respir Med 8(4):420-422

14. Ichimura T, Bonventre JV, Bailly V, Wei H, Hession CA, Cate RL et al (1998) Kidney injury molecule-1 (KIM-1), a putative epithelial cell adhesion molecule containing a novel immunoglobulin domain, is up-regulated in renal cells after injury. J Biol Chem 273(7):4135-4142

15. Han WK, Bailly V, Abichandani R, Thadhani R, Bonventre JV (2002) Kidney Injury Molecule-1 (KIM-1): a novel biomarker for human renal proximal tubule injury. Kidney Int 62(1):237-244

16. World Health Organization (2020) Clinical management of COVID-19: interim guidance, 27 May 2020. World Health Organization, Geneva

17. Kdigo A, Work Group (2012) KDIGO clinical practice guideline for acute kidney injury. Kidney Int Suppl 2(1):1-138

18. Levin A, Stevens PE, Bilous RW, Coresh J, De Francisco AL, De Jong PE et al (2013) Kidney Disease: Improving Global Outcomes (KDIGO) CKD Work Group. KDIGO 2012 clinical practice guideline for the evaluation and management of chronic kidney disease. Kidney Int Suppl 3(1):1-150

19. Santoriello D, Khairallah P, Bomback AS, Xu K, Kudose S, Batal I et al (2020) Postmortem kidney pathology findings in patients with COVID-19. J Am Soc Nephrol 31(9):2158-2167

20. Akilesh S, Nast CC, Yamashita M, Henriksen K, Charu V, Troxell ML et al (2021) Multicenter clinicopathologic correlation of kidney biopsies performed in COVID-19 patients presenting with acute kidney injury or proteinuria. Am J Kidney Dis 77(1):82-93.e1

21. Gross O, Moerer O, Weber M, Huber TB, Scheithauer S (2020) COVID-19-associated nephritis: early warning for disease severity and complications? Lancet 395(10236):e87-e88

22. Fisher M, Neugarten J, Bellin E, Yunes M, Stahl L, Johns TS et al (2020) AKI in hospitalized patients with and without COVID-19: a comparison study. J Am Soc Nephrol 31(9):2145-2157

23. Cheng Y, Luo R, Wang K, Zhang M, Wang Z, Dong L et al (2020) Kidney disease is associated with in-hospital death of patients with COVID-19. Kidney Int 97(5):829-838

24. Hirsch JS, Ng JH, Ross DW, Sharma P, Shah HH, Barnett RL et al (2020) Acute kidney injury in patients hospitalized with COVID19. Kidney Int 98(1):209-218

25. Yang X, Jin Y, Li R, Zhang Z, Sun R, Chen D (2020) Prevalence and impact of acute renal impairment on COVID-19: a systematic review and meta-analysis. Crit Care 24(1):356

26. Haase M, Kellum JA, Ronco C (2012) Subclinical AKI-an emerging syndrome with important consequences. Nat Rev Nephrol 8(12):735-739

27. Nickolas TL, Schmidt-Ott KM, Canetta P, Forster C, Singer E, Sise $M$ et al (2012) Diagnostic and prognostic stratification in the emergency department using urinary biomarkers of nephron damage: a multicenter prospective cohort study. J Am Coll Cardiol 59(3):246-255

28. Coca SG, Garg AX, Thiessen-Philbrook H, Koyner JL, Patel UD, Krumholz HM et al (2014) Urinary biomarkers of AKI 
and mortality 3 years after cardiac surgery. J Am Soc Nephrol 25(5):1063-1071

29. McWilliam SJ, Antoine DJ, Jorgensen AL, Smyth RL, Pirmohamed M (2018) Urinary biomarkers of aminoglycoside-induced nephrotoxicity in cystic fibrosis: kidney injury molecule- 1 and neutrophil gelatinase-associated lipocalin. Sci Rep 8(1):5094

30. Huang Y, Tian Y, Likhodii S, Randell E (2019) Baseline urinary KIM-1 concentration in detecting acute kidney injury should be interpreted with patient pre-existing nephropathy. Pract Lab Med. 15:e00118

31. Cai J, Jiao X, Luo W, Chen J, Xu X, Fang Y et al (2019) Kidney injury molecule-1 expression predicts structural damage and outcome in histological acute tubular injury. Ren Fail 41(1):80-87

32. Werion A, Belkhir L, Perrot M, Schmit G, Aydin S, Chen Z et al (2020) SARS-CoV-2 causes a specific dysfunction of the kidney proximal tubule. Kidney Int 98(5):1296-1307

33. Hong XW, Chi ZP, Liu GY, Huang H, Guo SQ, Fan JR et al (2020) Characteristics of renal function in patients diagnosed with COVID-19: an observational study. Front Med (Lausanne) 7:409
34. Mohamed M, Lukitsch I, Torres-Ortiz A, Walker J, Varghese V, Hernandez-Arroyo C et al (2019) Acute kidney injury associated with coronavirus disease 2019 in urban New Orleans. Kidney 360 1:614-622

35. Pei G, Zhang Z, Peng J, Liu L, Zhang C, Yu C et al (2020) Renal involvement and early prognosis in patients with COVID-19 pneumonia. J Am Soc Nephrol 31(6):1157-1165

36. Rubin S, Orieux A, Prevel R, Garric A, Bats ML, Dabernat S et al (2020) Characterization of acute kidney injury in critically ill patients with severe coronavirus disease 2019. Clin Kidney J 13(3):354-361

Publisher's Note Springer Nature remains neutral with regard to jurisdictional claims in published maps and institutional affiliations. 